\title{
Null alleles are ubiquitous at microsatellite loci in the Wedge Clam (Donax trunculus)
}

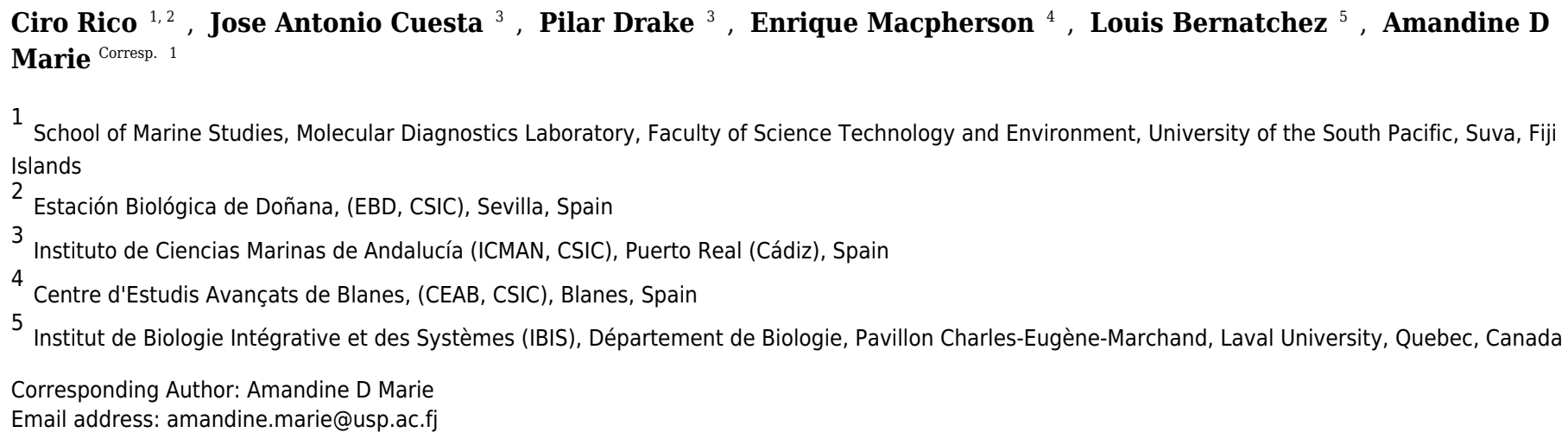

Recent studies have reported an unusually high frequency of nonamplifying alleles at microsatellite loci in bivalves. Null alleles have been associated with heterozygous deficits in many studies. While several studies have tested for its presence using different analytical tools, few have empirically tested for its consequences in estimating population structure and differentiation. We characterised 16 newly developed microsatellite loci and show that null alleles are ubiquitous in the wedge clam, Donax trunculus. We carried out several tests to demonstrate that the large heterozygous deficits observed in the newly characterised loci were most likely due to null alleles. We tested the robustness of microsatellite genotyping for population assignment by showing that well-recognised biogeographic regions of the south Atlantic and south Mediterranean coast of Spain harbour genetically different populations. 
1 Null alleles are ubiquitous at microsatellite loci in the Wedge Clam (Donax trunculus)

2

3 Ciro Rico $^{1,2} \S$, José Antonio Cuesta ${ }^{3}$, Pilar Drake $^{3}$, Enrique Macpherson $^{4}$, Louis Bernatchez ${ }^{5}$

$4 *$ Amandine D. Marie $^{1} \S$

5

$6 \quad{ }^{1}$ School of Marine Studies, Molecular Diagnostics Laboratory, Faculty of Science

7 Technology and Environment, The University of South Pacific, Suva, Fiji Islands

$8 \quad{ }^{2}$ Estación Biológica de Doñana, (EBD, CSIC), Sevilla 41092, Spain

$9{ }^{3}$ Instituto de Ciencias Marinas de Andalucía (ICMAN, CSIC), Puerto Real (Cádiz) 11519,

10 Spain

$11{ }^{4}$ Centre d'Estudis Avançats de Blanes, (CEAB, CSIC), Blanes 17300, Spain

$12{ }^{5}$ Institut de Biologie Intégrative et des Systèmes (IBIS), Département de Biologie, Université

13 Laval, Pavillon Charles-Eugène-Marchand, Québec G1V 0A6, Canada

14

15 *Corresponding author: Amandine D. Marie, amandine.marie@usp.ac.fj

16

17 §These authors have equally contributed to this work 


\section{Manuscript to be reviewed}

\section{ABSTRACT}

20 Recent studies have reported an unusually high frequency of nonamplifying alleles at

21 microsatellite loci in bivalves. Null alleles have been associated with heterozygous deficits in

22 many studies. While several studies have tested for its presence using different analytical

23 tools, few have empirically tested for its consequences in estimating population structure and

24 differentiation. We characterised 16 newly developed microsatellite loci and show that null

25 alleles are ubiquitous in the wedge clam, Donax trunculus. We carried out several tests to

26 demonstrate that the large heterozygous deficits observed in the newly characterised loci

27 were most likely due to null alleles. We tested the robustness of microsatellite genotyping for

28 population assignment by showing that the well-recognised biogeographic regions of the

29 south Atlantic and south Mediterranean Coast of Spain harbour genetically different

30 populations.

INTRODUCTION

37 Recent studies have reported an unusually high frequency of non-amplifying alleles, also

38 known as null alleles, at microsatellite loci in bivalves (Hargrove et al. 2015; Chiesa et al.

39 2016) including the wedge clam (Nantón et al. 2014). Null alleles occur when mutations in

40 the binding site of the targeted DNA sequence prevent the efficient annealing of at least one

41 primer resulting in failure of amplification during the PCR reaction. Null alleles can also

42 occur via the segmental aneuploidy, where one chromosome has a deletion containing the

43 primer binding site. For instance, in a study of allelic BAC sequences in the Pacific oyster 


\section{Manuscript to be reviewed}

44 obtained by the Oyster Genome Consortium, 42 of 101 microsatellite loci were found to

45 occur in the hemizygous state, owing to indels of various sizes (P. Gaffney, pers. com). Null

46 alleles can occur in homozygous state when the samples produce no amplification at all, and

47 in heterozygous state, when the sample appears as a homozygous individual for a particular

48 locus. Nantón et al. (2014), characterised the first nineteen polymorphic microsatellite

49 markers in D. trunculus to assist in the management of the species and allow the delineation

50 of conservation units essential in fisheries. Of the 19 loci characterised in this study, at least

5110 showed the presence of null alleles with frequencies ranging from 0.109 to 0.277 . Another

52 study aiming to examine the genetic variability and relationships between two Manila clam

53 (Ruditapes philippinarum) populations in Korea also suggested the occurrence of null alleles

54 in 7 loci out of 10 (Kim et al. 2014). More recently Chiesa et al. (2016) demonstrated the occurrence of null alleles though rigorous analytical tests in this species. The presence of null alleles has also been reported in a study that assessed the genetic diversity of four wild and six hatchery stocks of the hard clam (Mercenaria mercenaria) in Florida. Null alleles were present in all loci with frequencies ranging from 0.015 to 0.296 , but for 4 loci out of 7 , the frequency of null alleles was particularly high (>0.146) (Hargrove et al. 2015).

63 been associated with heterozygous deficits in many studies and several have tested their

64 presence and effects in population structure estimates using various analytical and simulation tools (e.g. Dąbrowski et al. 2015 and references therein). However, to the best of our

67 using population samples separated by well characterised biogeographic barriers. 


\section{Manuscript to be reviewed}

The wedge clam constitutes an important fishing resource in southern Spain and

69 Portugal due to its high economic value (Gaspar et al. 1999). In Europe alone, the recorded

70 landings in the last 12 years total 11,202 tons with a maximum yield of 1355 tons in 2005

71 (FAO-FIGIS 2016). Overall, the species has experienced a steady decline in recent years

72 reaching only 757 tons in 2014 (FAO-FIGIS 2016). Despite its overexploitation threat, $D$.

73 trunculus has not been studied from a population genetics perspective which could have

74 consequential implications for conservation (Hauser et al. 2008; Marie et al. 2016). Indeed,

75 understanding connectivity among populations through genetic structure provides tools to

76 determinate the appropriate units and spatial scale for fisheries conservation and management

77 (Waples \& Gaggiotti 2006; Funk et al. 2012). D. trunculus is an Atlantic-Mediterranean

78 warm-temperate species found in the Black Sea, in the Mediterranean Sea (Bayed \& Guillou

79 1985) and from Senegal to the northern Atlantic coast of France (Tebble 1966). It inhabits

sandy beaches exposed to tidal rhythms, characterised by intense wave action and sediment

81 instability (Brown \& McLachlan 1990). In these environments, populations are capable of

82 reaching sufficiently high densities to support large commercial fleets (Gaspar et al. 1999).

83 Because, wedge clams are filter feeders, they play important roles in the trophic structure of

84 beaches, but can also accumulate xenobiotic compounds making them, ideal model

85 organisms for environmental monitoring (Saavedra \& Bachere 2006). For this reason, they

86 may also constitute potential risks for human health when they are eaten and thus have been

87 extensively studied from an ecotoxicology perspective (Tlili et al. 2010; Yawetz et al. 2010;

88 Bouzas et al. 2011; Company et al. 2011; Hamdani \& Soltani-Mazouni 2011; Tlili et al.

89 2011).

Motivated by our interest in the conservation genetics of this important shellfish

91 resource, we used sequences generated by one NGS platform (Roche-454) for microsatellite

92 characterisation. Evidence of successful PCR amplification and allelic size variation across a 


\section{Manuscript to be reviewed}

93 subset of population samples allowed us to optimise 16 loci into 4 multiplex panels,

94 permitting the PCR amplification of several markers simultaneously. We then genotyped two

95 adjacent locations in the southern Atlantic Coast of Spain. One of them is situated within the

96 boundaries of the Doñana National Park, exploited, due to historic rights, only by artisanal

97 hand-operated dredges with restricted quotas. The second is approximately $85 \mathrm{~km}$ apart and is

98 exploited by a commercial boat-operated dredging fleet. We also genotyped a sample from

99 the south Mediterranean Coast of Spain. These samples, located on both sides of the Gibraltar

100 strait, which is a well-known major barrier to gene flow (Perez-Losada et al. 2007; Sá-Pinto

101 et al. 2012), allowed us to test the strength of the analyses using loci that have high

102 frequencies of null alleles in this species. Finally, we also show through empirical and

103 analytical tests that null alleles are ubiquitous in this species as previously suggested (Nantón 104 et al. 2014).

105

106

MATERIALS \& METHODS

107 Sampling

108 Samples of D. trunculus were collected from three sites, two located in the Atlantic coast and 109 one in the Mediterranean Sea. In the southwest Atlantic Coast of Spain, we collected samples 110 from two adjacent localities, Isla Canela $\left(\mathrm{n}=66 ; 37^{\circ} 10^{\prime} 11.334^{\prime \prime} \mathrm{N}, 7^{\circ} 22^{\prime} 14.25^{\prime \prime} \mathrm{W}\right)$ and

111 Doñana National Park $\left(\mathrm{n}=77 ; 36^{\circ} 55^{\prime} 6^{\prime \prime} \mathrm{N}, 6^{\circ} 28^{\prime} 20^{\prime \prime} \mathrm{W}\right)$, in the province of Huelva. The

112 third sample was obtained from the Mediterranean Coast of Spain from Caleta de Vélez,

113 Malaga $\left(\mathrm{n}=52 ; 36^{\circ} 44^{\prime} 49^{\prime \prime} \mathrm{N}, 4^{\circ} 4^{\prime} 32^{\prime \prime} \mathrm{W}\right)$. The Euclidean geographic distance between the 114 first 2 sites over coast line is $85 \mathrm{Km}$; while between the second and the third site is $287 \mathrm{Km}$

115 (128 Km from Doñana National Park to the Strait of Gibraltar and $158 \mathrm{Km}$ from there to the

116 third site). The first two samples are separated from the third one by the Strait of Gibraltar

117 which is well-recognised as a major geographical barrier to dispersal among marine taxa 
118 (Perez-Losada et al. 2007; Sá-Pinto et al. 2012). Permit of sampling was approved by the

119 Consejeria de Medio Ambiente (permit number 2011107300001463).

120 Library preparation and 454 GS-FLX pyrosequencing

121 DNA was extracted from different tissues using a slightly modified version of Aljanabi and

122 Martinez (1997) salting out method. Namely, after addition of the saline solution, the mixture

123 was centrifuged for $30 \mathrm{~min}$ at $10000 \mathrm{~g}$. Also, DNA precipitation was achieved by incubation

124 at $-20^{\circ} \mathrm{C}$ with $600 \mu \mathrm{L}$ isopropanol for $30 \mathrm{~min}$. Then, after washing out the pellet with $70 \%$

125 ethanol, we centrifuged at $10,000 \mathrm{~g}$ for $10 \mathrm{~min}$.

126 For the preparation of the SSR enriched library we followed the protocol of Santana et 127 al. (2009). In brief, we started with $20 \mu \mathrm{g}$ of high molecular weight genomic DNA (gDNA)

128 to enrich for SSR with ISSR-PCR method (Zietkiewicz et al. 1994), but without using the

129 final stage of cloning in a bacterial vector. The primers used were: ISSR1 (5'-DDB (GTC) 5-

130 3'), ISSR2 (5'-DHB (ATC) 5-3'), ISSR3 (5 'YHY (GT) 5G-3'), ISSR4 (5'-HVH (CAT) 5-3'),

131 ISSR5 (5'-NDB (TGT) 7C-3'), ISSR6 (5'-NDV (CT) 8-3'), and ISSR7 (5'-HBDB (AAC) 4-

$\left.1323^{\prime}\right)$. For each trial microsatellite-enriched DNA we analysed in $5 \mu \mathrm{g}$ in the Pyrosequencing

133 platform Roche 454 GS-FLX. For each sample, a single-lane sequencing run using portioned

134 sections of the PicoTiterPlate was performed according to the manufacturer's protocol

135 preparation. Sample preparation and analytical processing such as base calling, were also

136 performed on site according to the manufacturer's protocol.

\section{Contig assemblies and microsatellite detection}

138 To uncover sequences containing microsatellites in the pyrosequence reads, we used the

139 mreps software which has been designed to isolate and characterise highly polymorphic

140 microsatellite loci (Kolpakov et al. 2003). For the purpose of evaluating the number of

141 microsatellite-containing reads from the dataset, we defined 2 filtering steps. In the first step,

142 we included loci containing di- and tri-, nucleotides SSR with 20 to 60 base pairs of repeated 
143 units (i.e. at least 10 tandem repeats for di-, and 7 for tri-nucleotides), and a maximum

144 proportion of non-perfect repeats of 0.333.

145 Since hundreds of potential loci were detected in this filtering step and only loci with

146 sufficiently large flanking sequences around the microsatellite are potentially useful for

147 primer design for successful PCR amplification, we selected loci with a minimum of 40

148 flanking nucleotides on each side of the tandem repeated units. These loci are referred as

149 "Potentially Amplifiable Loci”, or PAL (Castoe et al. 2012). From the 85 PAL identified, we

150 selected 52 sequences for subsequent analyses. Primer design was performed using the on-

151 line version of Primer 3 (Rozen \& Skaletsky 2000). A total of 52 primer pairs were thus

152 designed and synthesised with the universal M13 primer in the 5' end of the forward primer

153 for their later analysis using a fluorescently labelled M13 primer according to Schuelke

154 (2000).

155 Multiplex optimisation

156 In the initial development phase, 8 individuals were used to verify the amplification of the

157 expected products for the 52 markers. PCR amplifications were performed in a reaction

158 volume of $15 \mu 1$, containing $15 \mathrm{ng}$ of gDNA, $0.5 \mathrm{pmol}$ of each primer, $75 \mu \mathrm{M}$ of each dNTP, $1591.5 \mathrm{mM}$ of $\mathrm{MgCl}_{2}, 1 \times$ PCR Green Master Mix buffer (Promega, Madison, WI USA) and 0.5

160 units of Taq polymerase (Promega). The PCR amplifications were done on an Applied

161 Biosystems 9700 DNA thermal cycler using the following conditions: an initial denaturation

162 step at $95^{\circ} \mathrm{C}$ for $10 \mathrm{~min}$ followed by 35 cycles of $30 \mathrm{~s}$ at $94^{\circ} \mathrm{C}, 1 \mathrm{~min}$ at either 58 or $50^{\circ} \mathrm{C}$,

163 depending on the primer's melting point, and $1 \min 30 \mathrm{~s}$ at $72^{\circ} \mathrm{C}$, and a last cycle of extension 164 at $72^{\circ} \mathrm{C}$ of $10 \mathrm{~min}$. The PCR conditions of successful amplifications were recorded for each

165 locus in order to make the subsequent multiplexing easier. The amplification products were

166 visualized by agarose gel electrophoresis using ethidium bromide staining. A total of 28

167 primer pairs amplified a clear PCR product and were selected for assessing their allelic size 
168 ranges using 48 individuals from the Doñana National Park. The rationale for using 48

169 samples is that by genotyping many, we aimed to detect a wide spectrum of the genetic

170 diversity present in the population and thus help in the subsequent design of the multiplex

171 panels. For this, we directly incorporated the fluorescently labelled M13 primer with

172 6FAM ${ }^{\mathrm{TM}}$ (Schuelke 2000), using the same PCR conditions described above. Polymorphism

173 and profile quality were first verified in simplex reactions. The PCR products were then

174 separated on a capillary sequencer (ABI 3130x Genetic Analyzer, Applied Biosystems, USA)

175 using GeneScan ${ }^{\mathrm{TM}} 500$ LIZ® Size Standard. Allele sizes were determined with the Gene

176 Mapper ${ }^{\circledR}$ V4.1 program (Applied Biosystems, USA). From this analysis, we identified 21 loci

177 that produced clear PCR products with relatively low levels of stuttering that were deemed of

178 sufficient quality for multiplexing. The forward primer of each of these loci was then

179 synthesised again, but this time incorporating in the 5 ' end one of the four fluorescent

180 markers $6 \mathrm{FAM}^{\mathrm{TM}}, \mathrm{VIC}^{\circledR}, \mathrm{NED}^{\mathrm{TM}}$ and $\mathrm{PET}^{\circledR}$ for multiplex PCR analysis.

181 We carried out a high number of tests to find optimal multiplexes, grouping several markers

182 of different allelic sizes in a single reaction and adjusting the concentration of the primers

183 according to the strength of the signal. For the multiplex reactions, we used the Qiagen ${ }^{\circledR}$

184 Multiplex PCR Kit (Qiagen, Canada). Final volumes and concentrations of the master mixes

185 were optimised to reduce the total genotyping cost (Guichoux et al. 2011). PCR reactions

186 were done in a final volume of $10 \mu \mathrm{L}$ for all multiplexes, with $3 \mu \mathrm{L}$ of primer mix, $5 \mu \mathrm{L}$ of

187 Qiagen Multiplex Mix, and $2 \mu \mathrm{L}$ of gDNA template $(10-15 \mathrm{ng} / \mu \mathrm{L})$. The cycling conditions

188 for the four multiplexes differed only in the annealing temperature (Table 1): an initial step at $18995^{\circ} \mathrm{C}$ for $15 \mathrm{~min}$; then 35 cycles at $94^{\circ} \mathrm{C}$ for $30 \mathrm{~s}, 54$ to $61^{\circ} \mathrm{C}$ for $3 \mathrm{~min}$ and $72^{\circ} \mathrm{C}$ for $1 \mathrm{~min}$; 190 and a final elongation step at $60^{\circ} \mathrm{C}$ for $30 \mathrm{~min}$. Only 16 loci produced codominant fragments

191 (i.e. one allele of maternal and one of paternal origin) in the 48 samples analysed. Table 1 
192 summarises the information about the primers sequences, concentrations in the primer premix

$193[\mu \mathrm{M}]$, GenBank accession numbers, and multiplex panel annealing temperatures.

\section{Genotype scoring and analyses}

195 Once multiplex conditions were optimised, we screened a total of 143 individuals from the

196 south Atlantic coast and 52 from the south Mediterranean coast of Spain for variation at 16

197 polymorphic microsatellite loci (Table 1: 16 loci). Several steps were taken to ensure the 198 consistency and accuracy of the genotype analysis and scoring. An initial allele size spread

199 table was developed from the genotypes of the 195 samples used in the multiplex PCR

200 amplifications for each locus. This table consisted of the observed size ranges for a given

201 allele in each locus, as determined by the GeneMapper ${ }^{\circledR}$ v4.1 (Applied Biosystems). The

202 table was further refined with the same set of samples genotyped in multiplex reactions and

203 then used to manually size each sample according to the scoring range. Afterwards, any

204 sample with a size value beyond the established range was reanalysed. In order to ensure that

205 the allele spread calibration held for each set of samples analysed, we always included the

206 same 6 individuals in each plate to be genotyped as reference standards. All genotype size

207 scores were checked twice to ensure size scoring accuracy. Furthermore, for each marker,

208 clear reading rules were defined and illustrated using screen shots of the chromatograms and

209 provided to a second, experienced scorer to ensure scoring consistency. A first estimate of

210 error rate was obtained by counting mismatches on the basis of these six positive controls

211 repeated two times in each multiplex panel. Positive controls were essential to include as they

212 allowed the verification of differences across sequencing runs. A second estimate of error rate

213 was obtained by comparing scoring across scorers. Two types of errors were distinguished.

214 Type A corresponds to cases where reader 1 called a genotype as heterozygous and reader 2

215 typed it as homozygous, or vice versa. Type B corresponds to cases where a different allele

216 was called by either of the scorers (Castoe et al. 2012). For each marker, we calculated the 
217 total number of alleles $\left(\mathrm{N}_{\mathrm{A}}\right)$, the observed $\left(\mathrm{H}_{\mathrm{O}}\right)$ and expected $\left(\mathrm{H}_{\mathrm{E}}\right)$ heterozygosities, the

218 polymorphism information content (PIC) and $F_{\text {IS }}$ using the program CERVUS 3.0

219 (Kalinowski et al. 2007) while and the null-allele frequencies (NAF) were determined using

220 the program ML-NULLFREQ (Kalinowski \& Taper 2006). Private alleles, defined as alleles

221 for a given locus present in only one of the two populations (South Atlantic Coast of Spain

222 and South Mediterranean Coast of Spain) were estimated using Convert version 1.31

223 (Glaubitz 2004). Samples were also tested for linkage disequilibrium and departure from

224 Hardy-Weinberg equilibrium by the Markov chain method using 100,000 dememorization

225 steps with the program Arlequin V 3.5 (Schneider et al. 2000; Excoffier et al. 2005). Finally,

226 we investigated whether there was any genetic differentiation between the two Atlantic

227 adjacent localities and subsequently between these and the Mediterranean sample using

228 weighted $\mathrm{F}_{\mathrm{ST}}(\theta)$ statistics by estimating the pairwise fixation index based on allele frequency

229 variation over all loci (Weir \& Cockerham 1984). The significance of genetic subdivision

230 was assessed using 1000 permutations in Arlequin.

231 We also estimated null allele frequencies and $F_{\text {ST }}$ using the software FreeNa (Chapuis \&

232 Estoup 2007) with a number of replicates fixed to 25,000. We ran this analysis using the

233 ENA correction method to efficiently correct for the positive bias induced by the presence of

234 null alleles on $F_{\mathrm{ST}}$ estimation and so, to provide an accurate estimation of $F_{\mathrm{ST}}$. We ran these

235 both software in order to see if the presence of high frequencies of null alleles can affect the

$236 \quad F_{\mathrm{ST}}$ estimates knowing that Arlequin does not take into account the presence of null alleles

237 compared to FreeNa.

238 Inbreeding, null allele estimations and genotype artefacts

239 The possibility of genotyping artefacts was examined in several ways. First, a subset of

240 samples $(\mathrm{N}=94)$ were re-amplified for each of the 16 loci under relaxed annealing

241 temperatures $\left(4^{\circ} \mathrm{C}\right.$ lower) to verify that additional alleles had not been missed. Second, we 
242 used the software MiCROCHECKER (van Oosterhout et al. 2004) to test for stuttering and large

243 allele drop-out and to obtain various estimates of the expected frequency of null alleles (r).

244 These, in turn, were used to calculate the expected number of null homozygotes (i.e. when

245 both alleles remain undetected) using the equation $4\left(\mathrm{Nr}^{2}\right)$ (Brookfield 1996) so that we could

246 compare these estimates to the actual number of individuals that fail to amplify

247 systematically in repeated PCR reactions. The analysis yielded a close match between the 248 expected and observed number of null homozygous. Third, we estimated the frequency of

249 null alleles using ML-NULLFREQ (Kalinowski \& Taper 2006) which is a maximum

250 likelihood estimator of the frequency of null alleles in a sample with or without missing data

251 which has been shown to be the single best-performing method (Dąbrowski et al. 2015).

252 Finally, to test the scoring's robustness and power of resolution to depict population genetic 253 structure of these microsatellite loci, we took advantage of the fact that it has extensively

254 been shown, in many marine taxa, that Atlantic populations are often genetically distinct

255 from Mediterranean populations (Patarnello et al. 2007). Therefore it is reasonable to

256 hypothesise that the samples from the Atlantic coast separated by $85 \mathrm{~km}$ of continuous

257 shoreline would not be genetically distinct from each other, but would be differentiated from 258 the Mediterranean population. To test this hypothesis even when the presence of null alleles 259 in our loci was already confirmed, we took advantage of the algorithm introduced by Falush 260 et al. (2007) in the program STRUCTURE V2.3.4 (Hubisz et al. 2009), which employs a 261 Bayesian clustering method to infer the most likely number of populations (K) assuming no a 262 priori structure and allows for the presence of null alleles in the dataset. To test the effects of 263 null alleles in the population structure depicted by these loci, we first estimated the most 264 likely number of populations (K) assuming no null alleles and using the LOCPRIOR option 265 and subsequently, we repeated the analysis but incorporating the RECESSIVEALLELES as 266 well as the LOCPRIOR options in STRUCTURE. In this case, the program assumes that the 


\section{Manuscript to be reviewed}

267 recessive allele is never observed in homozygous state but it might be present. This analysis,

268 partitions multilocus genotypes into clusters, while minimising departure from Hardy-

269 Weinberg expectations and linkage equilibrium among loci, and it estimates individual

270 ancestry proportions to each putative cluster. We therefore investigated the most likely K

271 running ten independent simulations. All simulations were run using default parameters in the

272 admixture model and with correlated allele frequencies. Each run included 100,000 iterations

273 of burnin, followed by 500,000 MCMC iterations used for parameter estimation. The most

274 likely value of $K$ was chosen using the delta $K(\Delta K)$ statistic (Evanno et al. 2005), based on

275 the rate of change between successive K values using the STRUCTURE HARVESTER

276 software (Earl \& vonHoldt 2012). The algorithm employed by this online program to

277 determine $\Delta \mathrm{K}$ by the Evanno method requires that at least three values of sequential $\mathrm{K}$ were

278 analysed. Thus, we ran ten independent simulations of $\mathrm{K}=1$ to 3 , despite knowing that the

279 maximum number of populations between Atlantic and Mediterranean samples is likely 2.

280 Then, potential admix individuals in each of the proposed clusters were identified using

281 posterior probabilities calculated for each individual in STRUCTURE.

Because of the high frequencies of null alleles, we also ran STRUCTURE with the

complete data set removing a different locus for each run using the same parameters as

described above (and including the RECESSIVEALLELES option) in order to identify the

level of information provided by each locus on the level of assignment. Then, we selected the

most informative loci and ran again STRUCTURE with and without the

287 RECESSIVEALLELES options still using the same parameters. We selected the most

288 informative loci from the run using the RECESSIVEALLELES option. For that, we created a

289 reference assignment's level from the average level of individuals' assignment of the South-

290 Atlantic Coast of Spain and the South-Mediterranean Coast of Spain respectively. If the

291 average level of individuals' assignment of a run (run when removing a locus) was under the 


\section{Manuscript to be reviewed}

292 reference one, then, we considered that the locus we removed for this run was very

293 informative.

294

\section{RESULTS AND DISCUSSION}

296 A total of 18,795 reads with an average length of $232 \mathrm{bp}$ were obtained from 454

297 pyrosequencing of a microsatellite enriched library and assembled into 595 contigs. We 298 identified 267 contigs $(44.9 \%)$ that contained microsatellite loci with at least 10 di- or 7 tri299 nucleotide repetitions. Of these, there were a total of 85 contigs $(31.8 \%)$ we identified as PAL 300 of di- or tri-nucleotide microsatellites and 182 contigs $(68.2 \%)$ as nonPAL (i.e. contigs 301 containing di- or trinucleotide microsatellites without suitable flanking PCR-primer sites).

302 Comparing across the two classes of repeats in PAL sequences, di-nucleotide repeats 303 represented $66.8 \%$ and tri-nucleotides $33.2 \%$.

From the microsatellite containing sequences, we selected 52 loci for testing their usefulness in multiplex genotyping and 37 produced PCR amplification when visualised on agarose gels and ethidium bromide staining. Of these 37 loci, 9 gave multiple bands, faint

307 bands, or fragments clearly defined but of a different size than the one expected from the 308 original sequence. These loci were discarded from subsequent analyses. Of the remaining 28 ,

30921 could be genotyped and displayed polymorphism in 48 individuals. These 21 loci were 310 deposited in GenBank under accession numbers HG792255.1 to HG792275.1. Five loci,

311 D.tru28, 30, 31, 39 and 46, amplified 2 or 3 loci simultaneously and were discarded from

312 further analyses. For the remaining loci, the number of alleles per locus ranged from 8 to 28 $313(\bar{\chi}=18.25, \sigma=5.86)$. Expected and observed heterozygosities, and polymorphic information

314 content ranged from 0.15 to $0.82(\bar{\chi}=0.53, \sigma=0.21), 0.54$ to $0.94(\bar{\chi}=0.81, \sigma=0.11)$, and 0.51 315 to $0.93(\bar{\chi}=0.79, \sigma=0.12)$, respectively. $F_{\text {IS }}$ estimates ranged from -0.07 to $0.74(\bar{\chi}=0.35$, $316 \sigma=0.04)$ (Table 1). Private alleles were present in all loci for the south Atlantic Coast of Spain 
317 and in 11 loci for the South Mediterranean Coast of Spain (Table S1). There was no evidence

318 of large allele dropout or genotyping errors due to stutter peaks for the 16 loci. However, all

319 loci but two (D.tru4 and D.tru16), showed a significant excess of homozygotes (deviation

320 from Hardy- Weinberg equilibrium, $P<0.001)$. There was no evidence of linkage

321 disequilibrium between any pair of loci. Genotyping was consistent across the 48 samples,

322 which were each tested twice yielding the same genotypes. The multiplex optimisation

323 yielded a total of 4 panels for the 16 primer pairs. The numbers of possible multiplex

324 combinations was limited due to the allele size ranges of the markers and differences in the

325 annealing temperatures of the primers. We were thus able to design two 3-plex, and two 5-

326 plex multiplex reactions after excluding the duplicated loci.

327 Before the advent of next-generation sequencing technologies, no genetic marker has

328 found such widespread use as microsatellites in the last two decades. This study joins an ever

329 increasing number of others that have successfully employed NGS to detect a virtually

330 unlimited number of polymorphic microsatellites across a wide variety of taxa (e.g. Wang et

331 al. 2012b; Wasimuddin et al. 2012; Whitney \& Karl 2012; Miller et al. 2013). Our study

332 takes advantage of Roche-454 titanium chemistry. Microsatellite-containing sequences from

333 shotgun and enriched libraries have also been reported across a broad range of taxa in recent

334 years (Angeloni et al. 2011; Santure et al. 2011; Wang et al. 2012a; Wang et al. 2012b;

335 Zhang et al. 2012). Together, these studies demonstrate that the main obstacle of costly de

336 novo isolation for using microsatellite markers for population studies and characterisation has

337 been rapidly overcome by the advent of NGS technologies. More importantly however, is the

338 fact that a growing proportion of research projects, working on modest financial resources,

339 are now capable of developing a suitable number of microsatellite markers at minimal

340 laboratory costs for virtually any taxa (Schoebel et al. 2013). The number of microsatellite

341 loci detected in this study illustrates how abundant these sequences are in eukaryotic 
342 genomes. Comparing the number of loci detected across studies and taxa is inherently

343 difficult because genomes vary substantially in their frequency of microsatellites and genome

344 size (Schoebel et al. 2013). However, the number of loci detected here favourably compares

345 with other studies (e.g. Wang et al. 2012a; Wang et al. 2012b; Zhang et al. 2012). For

346 example, in a recent study that used this technology, Schoebel et al. (2013) carried an

347 extensive survey of microsatellite abundance in 17 non-model species including plants, fungi,

348 invertebrates, birds and a mammals and determined if flanking regions were suitable for

349 primer development. They concluded that that depending on the species, a different amount

350 of 454 pyrosequencing data might be required for successful identification of a sufficient

351 number of microsatellite markers for ecological genetic studies. Irrespective of the difficulty

352 to compare microsatellite abundance in different taxa, an important message of our study is

353 that a virtually unlimited number of microsatellite loci can be identified from the large

354 amount of sequence data generated with NGS technologies. It is worth noticing as well that

355454 pyrosequencing has been replaced by other technologies which generally yield many

356 more sequences, but with shorter reads. The implications of these shorter reads may have an

357 important impact on marker development as microsatellites require the availability of long

358 flanking sequences for primer design. However, we have to consider that, despite the many

359 advantages of microsatellite markers, admittedly, the development of SNP markers by NGS

360 or SNP genotyping by sequencing have increasingly become attractive alternatives for many

361 reasons (e.g. availability of high numbers of annotated markers, low-scoring error rates,

362 improve genotyping results for poor quality samples, highly informative in terms of

363 segregation among populations, ability to examine neutral variation and regions under

364 selection, etc., (Brumfield et al. 2003; Rosenberg et al. 2003; Morin et al. 2004; Liu et al.

365 2005; Grewe et al. 2015). 
Once the multiplex panels were optimised, we genotyped a total of 195 individuals

367 from the three sampled locations. Table 1 summarises the genetic diversity found in each

368 locus for all the samples analysed. The initial uncorrected typing error rate was 0.0023 per

369 reaction or 0.0015 per allele. The frequency of single-locus genotypes missing in the data set

370 was $<0.05$. Adjacent allele scoring error analysis of the final corrected data set yielded no

371 significant deviations $\left(X^{2}<2, P>0.15\right)$, hinting that there were very few remaining scoring

372 errors. From individuals genotyped as controls in each plate, we calculated a mistyping rate

373 of 0.0019 per allele genotyped. Type A and type B error rates were 0.015 and 0.021 for

374 multiplex 1, 0.013 and 0.008 for multiplex 2, 0.026 and 0.051 for multiplex 3, and 0.009 and

3750.014 for multiplex 4 , and 0.0152 and 0.023 across all loci and were mainly caused by

376 excessive stuttering of some long alleles. Once again all loci but D.tru4 and D.tru16 showed a

377 significant excess of homozygotes (deviation from Hardy- Weinberg equilibrium, $P<0.001$ )

378 (Table 1). Exact tests for genotypic linkage disequilibrium confirmed the absence of physical

379 linkage among most loci $(P<0.05$ after Bonferroni correction).

380 The results of the re-amplification of a subsample of individuals under lower

381 stringency mostly confirmed the above findings. However, only 10 loci produced codominant

382 fragments (i.e. one allele of maternal and one of paternal origin) under relaxed annealing

383 temperatures (Table S1: Original Annealing Temperature, New Annealing Temperature).

384 Locus D.tru16, which initially had not deviated from HWE, amplified multiple loci and thus

385 could not be used for this comparison. Furthermore, two additional loci showed null allele

386 frequencies not different from zero (D.tru6 and D.tru23). This result suggests that non-

387 amplifying alleles can be detected by reducing the stringency of the annealing temperature.

388 For the remaining 8 loci, no differences were observed in the heterozygous deficit or the null

389 allele frequencies supporting the presence of null alleles in the wedge clam genome (Table 390 2). 
The fact that most loci had large heterozygous deficits was an intriguing result which

392 deserves a thorough discussion. The first considerations that need to be evaluated in addition

393 of the occurrence of null alleles, are the presence of large allele dropout. As well, the level of

394 stuttering hampers in all these loci the reliable scoring of alleles differing within one or two

395 repeated units. Another possible explanation could be the differences in allele size as reported

396 by previous studies that have found that loci with longer alleles tend to have higher dropout

397 rates than those with shorter alleles (Sefc et al. 2003; Buchan et al. 2005; Broquet et al.

398 2007). In our study, allele size ranges ranged from 60 to $24(\bar{\chi}=46.2, \sigma=12.12)$ and from 110

399 to $30(\bar{\chi}=54.7, \sigma=26)$ for the original and relaxed annealing temperatures respectively (Table

400 2). It is thus expected that the largest allele at a locus would amplify with less efficiency than

401 the shortest one as the latter would outcompete the former during amplifications (Sefc et al.

402 2003; Buchan et al. 2005; Broquet et al. 2007). This will artificially increase the frequency of

403 null alleles even in the absence of true null alleles.

404 The analysis of null allele frequencies per population using the maximum likelihood

405 estimates implemented in ML-NullFreq confirmed the presence of null alleles in all loci but

406 D.tru4 and D.tru16. For these loci, null-allele frequencies estimates ranged between $0 \%$ and

$40731.2 \%(\bar{\chi}=0.160, \sigma=0.098)$ and all loci but D.tru4, D.tru16 and D.tru23 had a null-allele

408 frequencies above 5\% (Table 3). Results from FreeNa showed lower null allele frequencies

409 for each locus (except for the locus D.tru19) than results obtained using ML-NullFreq. Null

410 allele frequencies estimates ranged between $0 \%$ and $29.3 \%(\bar{\chi}=0.149, \sigma=0.090$; Table 3$)$.

411 This high frequency of null alleles found in this study is consistent with many reports in other

412 bivalve species (Launey et al. 2002; Nantón et al. 2014; Chiesa et al. 2016). Clams are filter

413 feeders and thus, are prone to accumulate xenobiotic compounds (Tlili et al. 2010; Yawetz et

414 al. 2010; Bouzas et al. 2011; Company et al. 2011; Hamdani \& Soltani-Mazouni 2011; Tlili

415 et al. 2011). It is plausible that the mutagenic action of some pollutants such as heavy metals 
416 (e.g. mercury or cadmium) (Wong 1988) can be responsible for an increased mutation rate in

417 clams. This could be further investigated by comparing samples representing genetically

418 distinct populations where organisms are either heavily exposed to pollutants in beaches

419 adjacent to major urban areas or those occupying habitats in remote locations where the level

420 of pollution is likely to be low and far from industrial pollution.

421 As expected due to the absence of biogeographic barriers and the proximity of the

422 sampling sites in the south Atlantic coast of Spain, the fixation index between samples from

423 Isla Canela and Doñana National Park was not significantly different from zero (Arlequin:

$424 \quad F_{\mathrm{ST}}=0.0028-P>0.05 ;$ FreeNa: $F_{\mathrm{ST}}$ including ENA correction $\left.=0.0025\right)$ indicating

425 uninterrupted migration of larvae across the area. Furthermore, as predicted, the fixation

426 index between these two localities and the Mediterranean sample was significantly different

427 from zero (Arlequin: $F_{\mathrm{ST}}=0.033-P<0.001$; FreeNa: $F_{\mathrm{ST}}$ including ENA correction $=0.032$ ).

428 The STRUCTURE analysis found that the two populations from the south Atlantic coast of

429 Spain were genetically homogeneous while they were significantly different from the

430 Mediterranean population with the highest $\Delta \mathrm{K}$ value equal to the number of biogeographic

431 regions $(K=2)$ and the programme showed that the 10 independent runs from $K=1$ to $K=3$

432 produced consistent results whether or not the RECESSIVEALLELES option was used in the

433 analysis (Fig. 1). Calculation of the statistic $\Delta \mathrm{K}$ from the STRUCTURE runs indicated that

434 the two biogeographic regions analysed have clearly differentiated populations.

435 When running STRUCTURE with the complete data set without a different locus for

436 each run, our results also showed that three microsatellite loci were very informative (D.tru4,

437 D.tru29 and D.tru32; Fig. S1, Table S2). Indeed, compared to the reference average level of

438 individuals' assignment $(84.7 \% \pm 2.5$ and $90.6 \% \pm 1.7$ for the South Atlantic Coast of Spain

439 and the South Mediterranean Coast of Spain, respectively), without these three loci, the

440 average level of individuals' assignment dropped to $65.5 \% \pm 6.5,68.2 \% \pm 3.7$ and $77.9 \% \pm$ 


\section{Manuscript to be reviewed}

4412.6 in the South Atlantic Coast of Spain and to $38.2 \% \pm 5.4,89.5 \% \pm 2.2$ and $89.9 \% \pm 2.8$ in

442 South Mediterranean Coast of Spain, respectively when excluding either locus D.tru4,

443 D.tru29 or D.tru32. Surprisingly, two of these loci (D.tru29 and D.tru32) have large

444 frequencies of null alleles $(0.311 \pm 0.030$ and $0.228 \pm 0.027$ for the locus D.tru29 and D.tru32

445 respectively; Table 3) confirming that even when microsatellite loci display a large

446 frequencies of null alleles, they can be nevertheless useful to identify the genetic structure of

447 populations. This conclusion was also corroborated with the $F_{\mathrm{ST}}$ results using FreeNa and

448 Arlequin, which showed that the use of the null allele's correction option had no effect in the

$449 \quad F_{\mathrm{ST}}$ estimates.

450 To the best of our knowledge, it is unusual that $87.5 \%$ of newly developed loci are

451 affected by any form of genotyping errors or allele dropout during PCR due to the presence

452 of long alleles. Although, simulation studies suggest that null alleles with frequencies

453 between $5 \%$ and $8 \%$ should have only minor effects on classical estimates of population

454 differentiation (Chapuis \& Estoup 2007), in our case null allele frequencies will render all

455 these loci, but three, completely useless in population genetic analyses if the heterozygous

456 deficits were due to null alleles. The fact that we found panmixia between samples from

457 adjacent localities with no apparent barriers to gene flow and a well-defined structure for

458 populations separated by a well-characterised biogeographic barrier suggests that the large

459 heterozygous deficits we found, cannot be entirely due to null alleles or genotyping artefacts.

460 If this was the case, one would have expected significant, albeit artificial, genetic differences

461 between the two adjacent localities. Alternatively, it suggests that despite the presence of null

462 alleles, clearly differentiated populations can be detected, although levels of genetic diversity

463 are most likely underestimated. Admittedly however, results pertaining to genetic structure

464 presented here should be interpreted cautiously until they are corroborated by other

465 approaches, namely SNP genotyping. 


\section{Manuscript to be reviewed}

466

467 CONCLUSIONS

468 In conclusion, we have shown that null alleles at microsatellite loci are unusually abundant in

469 the wedge clam and that genotyping artefacts are unlikely to completely explain the large

470 heterozygous deficits observed in all population samples. We have also shown that the high

471 frequencies of null alleles observed at these loci do not appear to have a significant effect in

472 the population genetic parameters commonly assessed for microsatellite loci. The expected

473 population structure between Atlantic and Mediterranean samples was confirmed for the tests

474 carried out. Furthermore, the unusually high frequency of null alleles reported in the wedge

475 clam and in other bivalve species is worth investigating further. As mentioned above, it is

476 reasonable to hypothesise that pollutants may increase mutation rates in bivalves, resulting in

477 high frequencies of null allele. Considering the economic value of $D$. trunculus, additional

478 investigations are essential to validate this assumption.

479

480 ACKNOWLEDGMENTS

481 We are grateful for the support given, throughout the project, by the Coordination Office of

482 Donana National Park and the Consejeria de Agricultura, Pesca y Desarrollo Rural of the

483 Andalusian Government. C.R. also acknowledges M. I. Rico, F. Hiraldo Cano, J.J. Negro

484 Balmaseda, Eric Normandeau and Guillaume Cote for their support during his stay in

485 Canada. We gratefully acknowledge the Associate Editor and the two referees, Mariah Scott

486 and Patrick Gaffney, for their relevant comments which allowed us to considerably improve

487 the manuscript.

488

489 REFERENCES

490

Peer] reviewing PDF | (2016:10:13747:1:1:CHECK 26 Feb 2017) 
491

492

493

494

495

496

497

498

499

500

501

502

503

504

505

506

507

508

509

510

511

512

513

514

515

516

517

518

519

520

521

522

523

524

525

526

527

528

529

530

531

532

533

534

535

536

537

538

539

540
Aljanabi S.M. \& Martinez I. (1997) Universal and rapid salt-extraction of high quality genomic DNA for PCR-based techniques. Nucleic Acids Research 25, 4692-3.

Angeloni F., Wagemaker C.A.M., Jetten M.S.M., den Camp H.J.M.O., JanssenMegens E.M., Francoijs K.J., Stunnenberg H.G. \& Ouborg N.J. (2011) De novo transcriptome characterization and development of genomic tools for Scabiosa columbaria L. using next-generation sequencing techniques. Molecular Ecology Resources 11, 662-74.

Bayed A. \& Guillou J. (1985) A CONTRIBUTION TO THE STUDY OF POPULATIONS OF THE DONAX GENUS - THE POPULATION OF DONAXTRUNCULUS L (MOLLUSCA, BIVALVIA). Annales De L Institut Oceanographique 61, 139-47.

Bouzas A., Aguado D., Marti N., Manuel Pastor J., Herraez R., Campins P. \& Seco A. (2011) Alkylphenols and polycyclic aromatic hydrocarbons in eastern Mediterranean Spanish coastal marine bivalves. Environmental Monitoring and Assessment 176, 169-81.

Brookfield J.F.Y. (1996) A simple new method for estimating null allele frequency from heterozygote deficiency. Molecular Ecology 5, 453-5.

Broquet T., Ménard N. \& Petit E. (2007) Noninvasive population genetics: a review of sample source, diet, fragment length and microsatellite motif effects on amplification success and genotyping error rates. Conservation Genetics 8 , 249-60.

Brown A.C. \& McLachlan A. (1990) Ecology of sandy shores.

Brumfield R.T., Beerli P., Nickerson D.A. \& Edwards S.V. (2003) The utility of single nucleotide polymorphisms in inferences of population history. Trends in Ecology \& Evolution 18, 249-56.

Buchan J.C., Archie E.A., Van Horn R.C., Moss C.J. \& Alberts S.C. (2005) Locus effects and sources of error in noninvasive genotyping. Molecular Ecology Notes 5, 680-3.

Castoe T.A., Streicher J.W., Meik J.M., Ingrasci M.J., Poole A.W., de Koning A.P.J., Campbell J.A., Parkinson C.L., Smith E.N. \& Pollock D.D. (2012) Thousands of microsatellite loci from the venomous coralsnake Micrurus fulvius and variability of select loci across populations and related species. Molecular Ecology Resources 12, 1105-13.

Chapuis M.-P. \& Estoup A. (2007) Microsatellite Null Alleles and Estimation of Population Differentiation. Molecular Biology and Evolution 24, 621-31.

Chiesa S., Lucentini L., Freitas R., Nonnis Marzano F., Ferrari C., Filonzi L., Breda S., Minello F., Figueira E. \& Argese E. (2016) Null alleles of microsatellites for Manila clam Ruditapes philippinarum. Animal Genetics 47, 135-6.

Company R., Serafim A., Lopes B., Cravo A., Kalman J., Riba I., DelValls T.A., Blasco J., Delgado J., Sarmiento A.M., Nieto J.M., Shepherd T.J., Nowell G. \& Bebianno M.J. (2011) Source and impact of lead contamination on deltaaminolevulinic acid dehydratase activity in several marine bivalve species along the Gulf of Cadiz. Aquatic Toxicology 101, 146-54.

Dąbrowski M., Bornelöv S., Kruczyk M., Baltzer N. \& Komorowski J. (2015) 'True'null allele detection in microsatellite loci: a comparison of methods, assessment of difficulties and survey of possible improvements. Molecular Ecology Resources 15, 477-88.

Earl D.A. \& vonHoldt B.M. (2012) STRUCTURE HARVESTER: a website and program for visualizing STRUCTURE output and implementing the Evanno method. Conservation Genetics Resources 4, 359-61. 
541 Evanno G., Regnaut S. \& Goudet J. (2005) Detecting the number of clusters of individuals using the software structure: a simulation study. Molecular Ecology 14, 2611-20.

Excoffier L., Laval G. \& Schneider S. (2005) Arlequin (version 3.0): An integrated software package for population genetics data analysis. Evolutionary Bioinformatics 1, 47-50.

Falush D., Stephens M. \& Pritchard J.K. (2007) Inference of population structure using multilocus genotype data: dominant markers and null alleles. Molecular Ecology Notes 7, 574-8.

FAO-FIGIS (2016) Fisheries Global Information System.

Funk W.C., McKay J.K., Hohenlohe P.A. \& Allendorf F.W. (2012) Harnessing genomics for delineating conservation units. Trends Ecol Evol 27, 489-96.

Gaspar M.B., Ferreira R. \& Monteiro C.C. (1999) Growth and reproductive cycle of Donax trunculus L., (Mollusca : Bivalvia) off Faro, southern Portugal. Fisheries Research 41, 309-16.

Glaubitz J.C. (2004) convert: A user-friendly program to reformat diploid genotypic data for commonly used population genetic software packages. Molecular Ecology Notes 4, 309-10.

Grewe P.M., Feutry P., Hill P.L., Gunasekera R.M., Schaefer K.M., Itano D.G., Fuller D.W., Foster S.D. \& Davies C.R. (2015) Evidence of discrete yellowfin tuna (Thunnus albacares) populations demands rethink of management for this globally important resource. Scientific Reports 5, 16916.

Guichoux E., Lagache L., Wagner S., Chaumeil P., Leger P., Lepais O., Lepoittevin C., Malausa T., Revardel E., Salin F. \& Petit R.J. (2011) Current trends in microsatellite genotyping. Molecular Ecology Resources 11, 591-611.

Hamdani A. \& Soltani-Mazouni N. (2011) Changes in Biochemical Composition of the Gonads of Donax trunculus L. (Mollusca, Bivalvia) from the Gulf of Annaba (Algeria) in Relation to Reproductive Events and Pollution. Jordan Journal of Biological Sciences 4, 149-56.

Hargrove J.S., Sturmer L., Scarpa J. \& Austin J.D. (2015) Assessment of Genetic Diversity in Wild and Aquaculture Stocks ofMercenaria mercenariain Florida. Journal of Shellfish Research 34, 355-65.

Hauser L., Waples R.S. \& Carvalho G.R. (2008) Special Issue: Advances in Marine Fish and Fisheries Genetics. Fish and Fisheries 9, 331-2.

Hubisz M.J., Falush D., Stephens M. \& Pritchard J.K. (2009) Inferring weak population structure with the assistance of sample group information. Molecular Ecology Resources 9, 1322-32.

Kalinowski S.T. \& Taper M.L. (2006) Maximum likelihood estimation of the frequency of null alleles at microsatellite loci. Conservation Genetics 7, 991-5.

Kalinowski S.T., Taper M.L. \& Marshall T.C. (2007) Revising how the computer program cervus accommodates genotyping error increases success in paternity assignment. Molecular Ecology 16, 1099-106.

Kim E.M., An H.S., Kang J.H., An C.M., Dong C.M., Hong Y.K. \& Park J.Y. (2014) New polymorphic microsatellite markers for the Korean manila clam (Ruditapes philippinarum) and their application to wild populations. Genetics and Molecular Research 13, 8163-73.

Kolpakov R., Bana G. \& Kucherov G. (2003) mreps: efficient and flexible detection of tandem repeats in DNA. Nucleic Acids Research 31, 3672-8. 


\section{Manuscript to be reviewed}

589 Launey S., Ledu C., Boudry P., Bonhomme F. \& Naciri-Graven Y. (2002) Geographic

590

591

592

593

594

595

596

597

598

599

600

601

602

603

604

605

606

607

608

609

610

611

612

613

614

615

616

617

618

619

620

621

622

623

624

625

626

627

628

629

630

631

632

633

634

635

636

637

638
Structure in the European Flat Oyster (Ostrea edulis L.) as Revealed by Microsatellite Polymorphism. Journal of Heredity 93, 331-51.

Liu N., Chen L., Wang S., Oh C. \& Zhao H. (2005) Comparison of single-nucleotide polymorphisms and microsatellites in inference of population structure. BMC Genetics 6, S26.

Marie A.D., Lejeusne C., Karapatsiou E., Cuesta J.A., Drake P., Macpherson E., Bernatchez L. \& Rico C. (2016) Implications for management and conservation of the population genetic structure of the wedge clam Donax trunculus across two biogeographic boundaries. Scientific Reports 6, 39152.

Miller A.D., Good R.T., Coleman R.A., Lancaster M.L. \& Weeks A.R. (2013) Microsatellite loci and the complete mitochondrial DNA sequence characterized through next generation sequencing and de novo genome assembly for the critically endangered orange-bellied parrot, Neophema chrysogaster. Molecular Biology Reports 40, 35-42.

Morin P.A., Luikart G., Wayne R.K. \& the S.N.P.w.g. (2004) SNPs in ecology, evolution and conservation. Trends in Ecology \& Evolution 19, 208-16.

Nantón A., Arias-Pérez A., Méndez J. \& Freire R. (2014) Characterization of nineteen microsatellite markers and development of multiplex PCRs for the wedge clam Donax trunculus (Mollusca: Bivalvia). Molecular Biology Reports 41, 5351-7.

Patarnello T., Volckaert F.A.M.J. \& Castilho R. (2007) Pillars of Hercules: is the Atlantic-Mediterranean transition a phylogeographical break? Molecular Ecology 16, 4426-44.

Perez-Losada M., Nolte M.J., Crandall K.A. \& Shaw P.W. (2007) Testing hypotheses of population structuring in the Northeast Atlantic Ocean and Mediterranean Sea using the common cuttlefish Sepia officinalis. Molecular Ecology 16, 2667-79.

Rosenberg N.A., Li L.M., Ward R. \& Pritchard J.K. (2003) Informativeness of genetic markers for inference of ancestry. Am J Hum Genet 73.

Rozen S. \& Skaletsky H. (2000) Primer3 on the WWW for general users and for biologist programmers. Methods in molecular biology (Clifton, N.J.) 132, 36586.

Sá-Pinto A., Branco M.S., Alexandrino P.B., Fontaine M.C. \& Baird S.J.E. (2012) Barriers to Gene Flow in the Marine Environment: Insights from Two Common Intertidal Limpet Species of the Atlantic and Mediterranean. PLoS One 7, e50330.

Saavedra C. \& Bachere E. (2006) Bivalve genomics. Aquaculture 256, 1-14.

Santana Q.C., Coetzee M.P.A., Steenkamp E.T., Mlonyeni O.X., Hammond G.N.A., Wingfield M.J. \& Wingfield B.D. (2009) Microsatellite discovery by deep sequencing of enriched genomic libraries. Biotechniques 46, 217-23.

Santure A.W., Gratten J., Mossman J.A., Sheldon B.C. \& Slate J. (2011) Characterisation of the transcriptome of a wild great tit Parus major population by next generation sequencing. BMC Genomics 12.

Schneider S., Roessli D. \& Excoffier L. (2000) Arlequin Ver. 2.0: A software for population genetics data analysis. Genetics and Biometry Laboratory, University of Geneva, Switzerland.

Schoebel C.N., Brodbeck S., Buehler D., Cornejo C., Gajurel J., Hartikainen H., Keller D., Leys M., Ricanova S., Segelbacher G., Werth S. \& Csencsics D. (2013) Lessons learned from microsatellite development for nonmodel 
639

640

641

642

643

644

645

646

647

648

649

650

651

652

653

654

655

656

657

658

659

660

661

662

663

664

665

666

667

668

669

670

671

672

673

674

675

676

677

678

679

680

681

682

683

684

685

686

687

organisms using 454 pyrosequencing. Journal of Evolutionary Biology 26, 1 12.

Schuelke M. (2000) An economic method for the fluorescent labeling of PCR fragments. Nature Biotechnology 18, 233-4.

Sefc K.M., Payne R.B., Sorenson M.D. \& Fleischer R.C. (2003) Microsatellite amplification from museum feather samples: effects of fragment size and template concentration on genotyping errors. The Auk 120, 982-9.

Tebble N. (1966) British bivalve shells. A handbook for identification.

Tlili S., Metais I., Ayache N., Boussetta H. \& Mouneyrac C. (2011) Is the reproduction of Donax trunculus affected by their sites of origin contrasted by their level of contamination? Chemosphere 84, 1362-70.

Tlili S., Metais I., Boussetta H. \& Mouneyrac C. (2010) Linking changes at subindividual and population levels in Donax trunculus: Assessment of marine stress. Chemosphere 81, 692-700.

van Oosterhout C., Hutchinson W.F., Wills P.M. \& Shipley P. (2004) MICROCHECKER: software for identifying and correcting genotyping errors in microsatellite data. Molecular Ecology Notes 4, 535-8.

Wang B., Ekblom R., Castoe T.A., Jones E.P., Kozma R., Bongcam-Rudloff E., Pollock D.D. \& Hoglund J. (2012a) Transcriptome sequencing of black grouse (Tetrao tetrix) for immune gene discovery and microsatellite development. Open Biology 2.

Wang R., Li C., Stoeckel J., Moyer G., Liu Z. \& Peatman E. (2012b) Rapid development of molecular resources for a freshwater mussel, Villosa lienosa (Bivalvia: Unionidae), using an RNA-seq-based approach. Freshwater Science 31, 695-708.

Waples R.S. \& Gaggiotti O. (2006) What is a population? An empirical evaluation of some genetic methods for identifying the number of gene pools and their degree of connectivity. Mol Ecol 15, 1419-39.

Wasimuddin, Cizkova D., Ribas A., Pialek J., de Bellocq J.G. \& Bryja J. (2012) Development and characterization of multiplex panels of microsatellite markers for Syphacia obvelata, a parasite of the house mouse (Mus musculus), using a high throughput DNA sequencing approach. Molecular and Biochemical Parasitology 185, 154-6.

Weir B.S. \& Cockerham C.C. (1984) Estimating F-statistics for the analysis of population structure. Evolution 38, 1358-70.

Whitney J.L. \& Karl S.A. (2012) Development of 38 microsatellite loci from the Arceye hawkfish, Paracirrhites arcatus, using next-generation sequencing and cross-amplification in other Cirrhitid species. Conservation Genetics Resources 4, 549-53.

Wong P. (1988) Mutagenicity of heavy metals. Bulletin of environmental contamination and toxicology 40, 597-603.

Yawetz A., Fishelson L., Bresler V. \& Manelis R. (2010) Comparison of the effects of pollution on the marine bivalve Donax trunculus in the vicinity of polluted sites with specimens from a clean reference site (Mediterranean Sea). Marine Pollution Bulletin 60, 225-9.

Zhang J., Liang S., Duan J., Wang J., Chen S., Cheng Z., Zhang Q., Liang X. \& Li Y. (2012) De novo assembly and Characterisation of the Transcriptome during seed development, and generation of genic-SSR markers in Peanut (Arachis hypogaea L.). BMC Genomics 13. 
688 Zietkiewicz E., Rafalski A. \& Labuda D. (1994) Genome fingerprinting by simple 689 sequence repeat (SSR)-anchored polymerase chain-reaction amplification. $690 \quad$ Genomics 20, 176-83.

691

692

693

694

695

696

697

698

699

700

701

702

703

704

705

706

707

708

709

710

711

712

713

714 


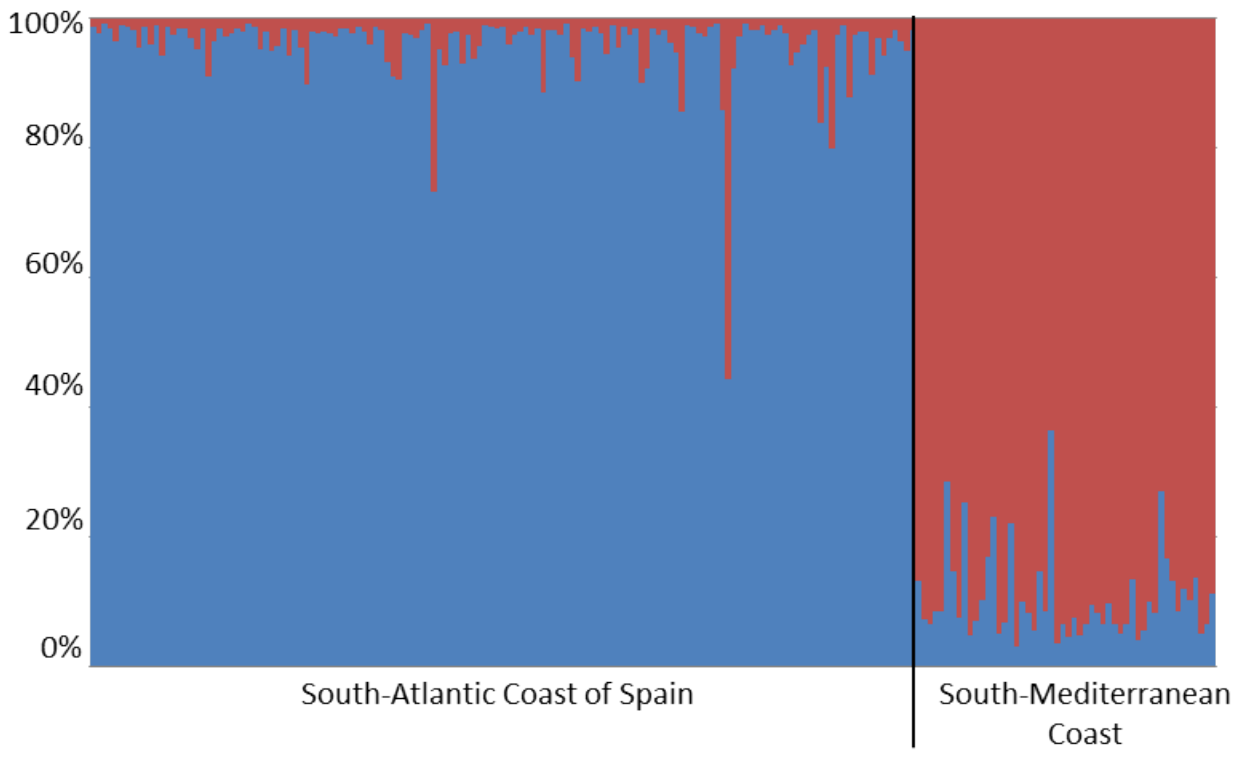

717 B

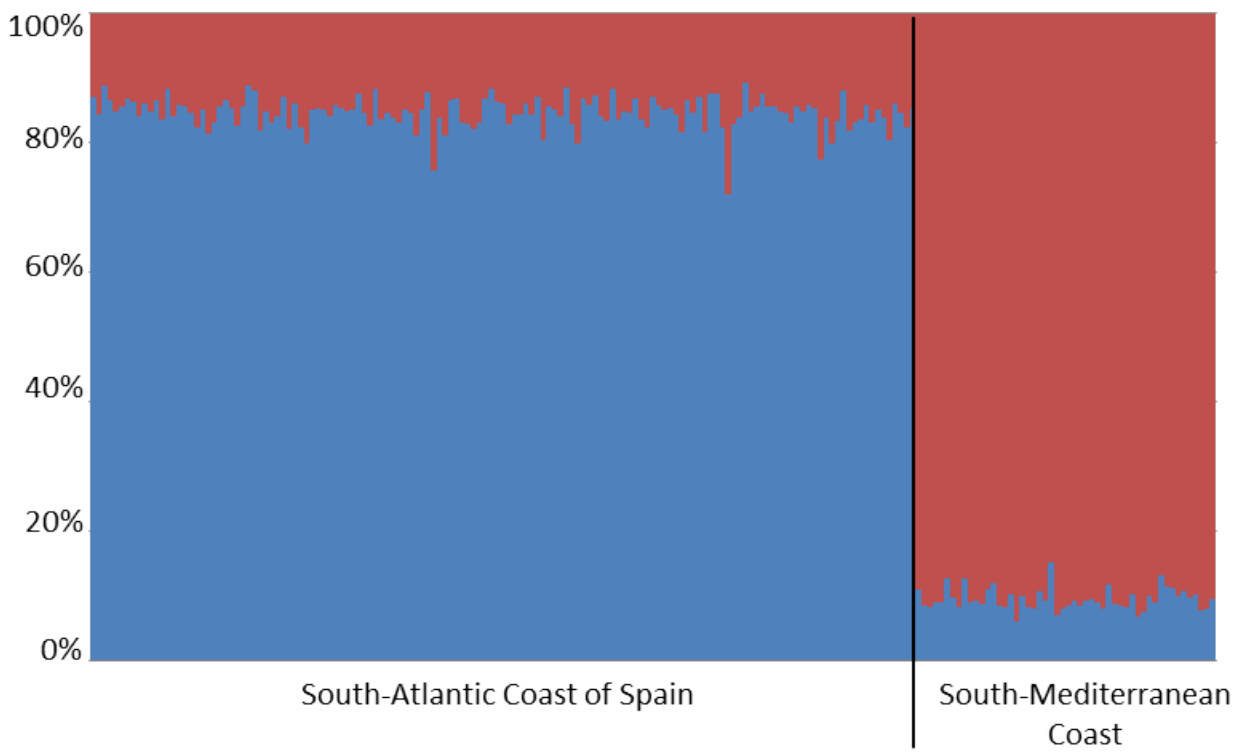

719 Figure 1: Clustering analysis in STRUCTURE considering information about population of

720 origin $(\mathrm{k}=2)$. Individuals are represented as vertical bars, where the amount of each colour

721 indicates the proportion of each inferred cluster. A represents the clusters obtained in the

722 simulations considering the absence of null alleles while B represents those assuming them.

723 Isla Canela and Doñana are represented by the label "South-Atlantic Coast of Spain", and

724 Caleta de Vélez by the label "South-Mediterranean Coast". 
725 Table 1: Details of the 16 microsatellite markers developed and optimized for multiplex. One hundred ninety five individuals were used for this 726 analysis. Locus identity, type of florescence dye in the forward primer, panel numbers (MP) indicate groupings of loci sharing a multiplex in the

727 PCR reaction, primer sequences, repeat motif SSR, primer concentrations $([\mathrm{C}] \mu \mathrm{M})$ in the primer mix, number of alleles $\left(\mathrm{N}_{\mathrm{A}}\right)$ found, range of

728 allele sizes (Range, bp), observed $\left(\mathrm{H}_{\mathrm{O}}\right)$ and expected $\left(\mathrm{H}_{\mathrm{E}}\right)$ heterozygosity, $F_{\mathrm{IS}}$, departure from Hardy-Weinberg equilibrium $(\mathrm{HWE})$,

729 polymorphism information content (PIC), and GenBank accession numbers. Annealing temperatures for each panel were as follows: Kit $3=$

$73060^{\circ} \mathrm{C}$, Kits $1,2,4=58^{\circ} \mathrm{C}$.

\begin{tabular}{|c|c|c|c|c|c|c|c|c|c|c|c|c|c|}
\hline $\begin{array}{l}\text { Locus } \\
\text { ID }\end{array}$ & Fluoro & MP & Forward Primer & Reverse Primer & SSR & $\begin{array}{l}{[C]} \\
\mu M\end{array}$ & $\mathrm{~N}_{\mathrm{A}}$ & Range & $\mathrm{H}_{\mathrm{O}}$ & $\mathrm{H}_{\mathrm{E}}$ & $F_{\text {IS }}$ & $\mathrm{PIC}$ & $\begin{array}{c}\text { GenBank } \\
\text { Acc. № }\end{array}$ \\
\hline D.tru2 & 6-FAM & 4 & ATTCTCCTACGGAGGGGCTA & GCGATGATTTCCTCCGTAAA & (ACA) & 3 & 17 & $97-148$ & 0.63 & 0.84 & 0.247 & 0.83 & HG792255 \\
\hline D.tru4 & 6-FAM & 3 & TGCACTTATAATCAACCGGAAG & CTTCCAGCAACACCACGTC & (TG) & 3.5 & 12 & $151-177$ & 0.69 & 0.75 & -0.069 & 0.71 & HG792256 \\
\hline D.tru6 & PET & 3 & GTTTTCTCACAGGCGTTCG & GCAGTGATAGGGTTAACGTATTTG & (CA) & 3.5 & 23 & $69-131$ & 0.59 & 0.85 & 0.316 & 0.84 & HG792257 \\
\hline D.tru8 & PET & 4 & AATATATTGCAGGCTGGTAGGG & TAAAATTGCCATGCGTGCAG & (ATC) & 3 & 11 & $127-157$ & 0.63 & 0.81 & 0.206 & 0.78 & HG792258 \\
\hline D.tru11 & 6-FAM & 2 & AGAACCTGATGTGCTGTGGA & CACGTTAGTACAAAGACCCTTTCC & $(\mathrm{GT})$ & 1 & 21 & $101-145$ & 0.57 & 0.79 & 0.268 & 0.78 & HG792259 \\
\hline D.tru14 & NED & 4 & TTTTTGTTCTTCTGAATAGTGCAA & TCGCCATCTTTTGTTGTTGT & (AA) & 3 & 14 & $75-129$ & 0.15 & 0.54 & 0.738 & 0.51 & HG792260 \\
\hline D.tru15 & 6-FAM & 1 & TGTCACTAATACAGGATTTCTCACG & AATAGCATCTCTCACACAGACACA & $(\mathrm{AC})$ & 5 & 27 & $219-279$ & 0.62 & 0.93 & 0.332 & 0.92 & HG792261 \\
\hline D.tru16 & VIC & 3 & TGCTCCTTATCATTTCAATTGTG & TGCAAACCATCTTCTGGTTG & $(\mathrm{AC})$ & 4.5 & 20 & $70-114$ & 0.82 & 0.90 & 0.107 & 0.89 & HG792262 \\
\hline D.tru19 & VIC & 1 & AACACCCATAGCGACGAAAA & GATGACCTGTGAATACATGAAGGA & $(\mathrm{AC})$ & 1.5 & 23 & $126-180$ & 0.73 & 0.94 & 0.214 & 0.93 & HG792263 \\
\hline D.tru22 & PET & 1 & TGAAGACATGGCAAAATCCA & TGAGCATATTTCTCTTTCGTAGG & (TTG) & 3 & 8 & $218-245$ & 0.27 & 0.69 & 0.601 & 0.64 & HG792264 \\
\hline D.tru23 & NED & 1 & CAAGCACGTTAGACAAAGTCC & ACCTGATGTGTTGTGGACGA & $(\mathrm{AC})$ & 1 & 28 & $105-167$ & 0.73 & 0.84 & 0.123 & 0.83 & HG792265 \\
\hline D.tru26 & NED & 2 & TGGAGGTAATTAGATGGTCCAG & ACGCTGGCATCGTTCTCTAT & $(A G)$ & 0.8 & 22 & $57-119$ & 0.65 & 0.92 & 0.297 & 0.91 & HG792266 \\
\hline D.tru29 & VIC & 2 & TGAATTTAGTGATTGGCAAAGCTA & ACGGGTGGCATACAACTTGA & (TGT) & 4 & 20 & $262-322$ & 0.31 & 0.88 & 0.638 & 0.87 & HG792268 \\
\hline D.tru32 & VIC & 4 & CCGAATGTCCCTTTTGTTGT & TGGGTCCTGGAGGGTAAAAT & (TTG) & 3 & 18 & $196-281$ & 0.28 & 0.66 & 0.572 & 0.64 & HG792271 \\
\hline D.tru40 & PET & 1 & GACATTAAGGAGTGGTTGCGTA & CATCAACCGAAAACTCTATAAACTG & (TG) & 1 & 17 & $119-155$ & 0.60 & 0.88 & 0.304 & 0.87 & HG792273 \\
\hline D.tru49 & NED & 4 & GAGTATTTCTAACGGTCTTCAAGTTAT & GCATTTATCTTATGTGGTGTTTGC & $(\mathrm{CA})$ & 3 & 11 & 148-182 & 0.24 & 0.78 & 0.663 & 0.74 & HG792275 \\
\hline
\end{tabular}


731 Table 2: Characteristics of the 10 microsatellite markers with original (OA) and new (NA) annealing temperature for 94 individuals (Isla Canela: 732 27; Doñana: 37; Caleta de Vélez: 30) including the number of alleles $\left(\mathrm{N}_{\mathrm{A}}\right)$, range of allele sizes (Range, bp), observed $\left(\mathrm{H}_{\mathrm{O}}\right)$ and expected $\left(\mathrm{H}_{\mathrm{E}}\right)$ 733 heterozygosity, polymorphism information content (PIC) and null-allele frequency (NAF).

734

\begin{tabular}{l|cc|cc|cc|cc|cc|cc|} 
Locus & \multicolumn{3}{|c}{$\mathbf{N}_{\mathbf{A}}$} & \multicolumn{2}{|c|}{ Range } & \multicolumn{2}{|c|}{$\mathbf{H}_{\mathbf{O}}$} & \multicolumn{2}{|c|}{$\mathbf{H}_{\mathbf{E}}$} & \multicolumn{2}{|c|}{ PIC } & \multicolumn{2}{c|}{ NAF } \\
& $\mathbf{O A}$ & $\mathbf{N A}$ & $\mathbf{O A}$ & $\mathbf{N A}$ & $\mathbf{O A}$ & $\mathbf{N A}$ & $\mathbf{O A}$ & $\mathbf{N A}$ & $\mathbf{O A}$ & NA & OA & NA \\
\hline D.tru4 & 11 & 34 & $151-179$ & $99-209$ & 0.641 & 0.809 & 0.759 & 0.930 & 0.718 & 0.920 & 0.093 & 0.065 \\
D.tru6 & 18 & 26 & $71-131$ & $69-161$ & 0.527 & 0.923 & 0.842 & 0.882 & 0.821 & 0.868 & 0.239 & -0.030 \\
D.tru11 & 20 & 19 & $101-145$ & $111-149$ & 0.581 & 0.723 & 0.807 & 0.820 & 0.792 & 0.801 & 0.173 & 0.056 \\
D.tru15 & 25 & 23 & $219-279$ & $219-281$ & 0.670 & 0.759 & 0.928 & 0.936 & 0.918 & 0.926 & 0.158 & 0.101 \\
D.tru19 & 22 & 20 & $126-176$ & $128-170$ & 0.734 & 0.841 & 0.937 & 0.937 & 0.928 & 0.927 & 0.120 & 0.052 \\
D.tru22 & 7 & 8 & $221-245$ & $215-245$ & 0.233 & 0.333 & 0.658 & 0.692 & 0.594 & 0.636 & 0.480 & 0.342 \\
D.tru23 & 22 & 22 & $107-159$ & $107-151$ & 0.702 & 0.886 & 0.838 & 0.864 & 0.823 & 0.850 & 0.093 & -0.020 \\
D.tru26 & 19 & 21 & $57-103$ & $67-111$ & 0.696 & 0.783 & 0.924 & 0.934 & 0.913 & 0.925 & 0.140 & 0.086 \\
D.tru29 & 17 & 13 & $268-322$ & $265-310$ & 0.344 & 0.203 & 0.872 & 0.851 & 0.855 & 0.828 & 0.434 & 0.611 \\
D.tru40 & 17 & 18 & $119-163$ & $121-161$ & 0.596 & 0.830 & 0.866 & 0.885 & 0.849 & 0.869 & 0.182 & 0.030
\end{tabular}

735

736 
741 Table 3: Estimate of null allele frequencies per locus and populations. The maximum likelihood and the ENA correction methods were used,

742 using ML-NullFreq and FreeNa respectively.

743

\begin{tabular}{|l|cc|cc|cc|cc|cc|}
\cline { 2 - 9 } \multicolumn{1}{c|}{} & \multicolumn{2}{c|}{ Isla Canela } & \multicolumn{2}{c|}{ Doñana } & \multicolumn{2}{c|}{ Caleta de Vélez } & \multicolumn{2}{c|}{ Mean } & \multicolumn{2}{c|}{ SD } \\
\hline Locus & $\begin{array}{c}\text { ML- } \\
\text { ID }\end{array}$ & FreeNa & $\begin{array}{c}\text { ML- } \\
\text { NullFreq }\end{array}$ & FreeNa & $\begin{array}{c}\text { ML- } \\
\text { NullFreq }\end{array}$ & FreeNa & $\begin{array}{c}\text { ML- } \\
\text { NullFreq }\end{array}$ & FreeNa & $\begin{array}{c}\text { ML- } \\
\text { NullFreq }\end{array}$ & FreeNa \\
\hline D.tru2 & 0.168 & 0.168 & 0.106 & 0.106 & 0.107 & 0.09 & 0.127 & 0.121 & 0.036 & 0.041 \\
D.tru4 & 0 & 0 & 0 & 0 & 0 & 0 & 0 & 0 & 0 & 0 \\
D.tru6 & 0.106 & 0.106 & 0.112 & 0.112 & 0.222 & 0.173 & 0.147 & 0.131 & 0.065 & 0.037 \\
D.tru8 & 0.144 & 0.114 & 0.125 & 0.125 & 0.05 & 0.044 & 0.106 & 0.095 & 0.05 & 0.044 \\
D.tru11 & 0.113 & 0.113 & 0.139 & 0.117 & 0.084 & 0.084 & 0.112 & 0.105 & 0.028 & 0.018 \\
D.tru14 & 0.307 & 0.264 & 0.356 & 0.238 & 0.273 & 0.273 & 0.312 & 0.259 & 0.042 & 0.018 \\
D.tru15 & 0.199 & 0.16 & 0.189 & 0.164 & 0.164 & 0.142 & 0.184 & 0.155 & 0.018 & 0.011 \\
D.tru16 & 0.014 & 0.014 & 0.033 & 0.033 & 0.083 & 0.083 & 0.043 & 0.043 & 0.036 & 0.036 \\
D.tru19 & 0.088 & 0.088 & 0.111 & 0.111 & 0.102 & 0.102 & 0.1 & 0.101 & 0.012 & 0.012 \\
D.tru22 & 0.3 & 0.282 & 0.263 & 0.248 & 0.23 & 0.204 & 0.264 & 0.245 & 0.035 & 0.039 \\
D.tru23 & 0.038 & 0.038 & 0.064 & 0.064 & 0.008 & 0.008 & 0.037 & 0.037 & 0.028 & 0.028 \\
D.tru26 & 0.09 & 0.09 & 0.165 & 0.165 & 0.204 & 0.165 & 0.153 & 0.14 & 0.058 & 0.043 \\
D.tru29 & 0.277 & 0.277 & 0.322 & 0.322 & 0.333 & 0.28 & 0.311 & 0.293 & 0.03 & 0.025 \\
D.tru32 & 0.258 & 0.258 & 0.219 & 0.219 & 0.206 & 0.206 & 0.228 & 0.228 & 0.027 & 0.027 \\
D.tru40 & 0.103 & 0.103 & 0.223 & 0.223 & 0.09 & 0.09 & 0.139 & 0.139 & 0.073 & 0.073 \\
D.tru49 & 0.299 & 0.299 & 0.359 & 0.335 & 0.222 & 0.222 & 0.293 & 0.285 & 0.069 & 0.058 \\
\hline
\end{tabular}

744

745 SD: standard deviation 